\title{
AN OVERVIEW OF EPIGENETIC DRUGS, AND THEIR VIRTUAL SCREENING STUDY RETRIEVED FROM ZINC DATABASE ALONG WITH AN AUTODOCK STUDY OF THE BEST INHIBITOR
}

\section{EIICHI AKAHO*}

\author{
Faculty of Pharmaceutical Sciences, Kobe Gakuin Univercity, 1-1-3 Minatojima, Chuo-ku, Kobe, 6508586 Japan
}

*Email: akaho@pharm.kobegakuin.ac.jp

Received: 02 Jun 2021, Revised and Accepted: 28 Jul 2021

\begin{abstract}
Objective: Over the last 30 y cancer epigenetics research has grown extensively. It is note-worthy to recognize that epigenetic misregulation could substantiate the development of cancer and we need to continue to look for anti-neoplastic epi-drugs. Taking into consideration this phenomenon, our first aim is to search for an effective epi-drugs by virtual screening from ZINC database and to explore the validity of the virtual screening. The second aim is to explore a binding conformation of the top affinity ligands against macromolecules, by docking experiment.
\end{abstract}

Methods: The virtual screening was conducted by our Virtual Screening by Docking (VSDK) algorithm and procedure. Small molecules were randomly downloaded by ZINC database. For docking experiment, AutoDock 4.2.6 and AutoDock Tool were used.

Results: It took eight to ten hours for the successful virtual screening of the 2778 small compounds retrieved at random from ZINC database. Among histone H2B E76K mutant (HHEM) inhibitors and DNA methyltransferase (DNMT) inhibitors, the first ranked inhibitors were 1H-1,2,4triazole-3,5-diamine and 2-ethyl-1,3,4-oxadiazole respectively.

Conclusion: As for the molecular structures obtained from virtual screening, most of the top ten HHEM and DNMT inhibitors contained 5-member rings. More than two times in affinity difference between the top and bottom ten compounds would indicate a successful virtual screening experiment. The histogram chart of AutoDock4 runs appeared in the lowest affinity region with two or three hydrogen bonds indicating a reliable conformation docking.

Keywords: Epigenetic drugs, Virtual screening, AutoDock, Hydrogen bond, Histogram, Cluster, Histone H3B E6K mutant, DNA methyltransferase

(C) 2021 The Authors. Published by Innovare Academic Sciences Pvt Ltd. This is an open access article under the CC BY license (https://creativecommons.org/licenses/by/4.0/) DOI: https://dx.doi.org/10.22159/ijap.2021v13i5.42275. Journal homepage: https://innovareacademics.in/journals/index.php/ijap

\section{INTRODUCTION}

The global burden of cancer, a disease responsible for nearly 10 million deaths per year, is predicted to top 13 million deaths by 2030 in the world. The cancer mortality rate rose until 1991, then fell every year through 2017 , resulting in $29 \%$ overall decline. We believe that chemotherapeutic advancement, one of the major cancer treatment strategies, has made a great contribution for this progress [1-4].

Cancer epigenetics and related fields have been intensely studied over 30 y [5-17[. The epigenetic regulation comprises histone modifications including acetylation, deacetylation, phosphorylation and methylation, DNA hydroxy-methylation and its oxidation derivative production, nucleosome remodeling, and finally RNAmediated targeting, A substantial number of biological processes fundamental to the genesis of cancer are regulated by epigenetics. It was reported and highlighted that epigenetic misregulation could culminate in cancer development [5-18]

The dynamic regulation of chromatin involves ATP-dependent nucleosome-remodeling complexes which can be classified into the four families; a) the switching/sucrose non-fermenting family, b)the imitation switch family, c) chromodomain helicase DNA-binding protein family, and d) the inositol requiring 80 (INO80) family [19, 20].

Bromodomain (BRD) is evolutionarily preserved protein-protein interaction modules that are observed in various proteins with diverse catalytic and scaffolding functions and are present in most tissues. Bromodomains selectively bind to acetylated lysine residues, particularly in histones. Dysregulations of bromodomain-containing proteins are frequently found in cancer. Bromodomain protein 4 (BRD4) is a chromatin-binding protein implicated in cancer which functions as a scaffold for transcription factors at promoters and enhancers [21-27].

Glycosylation is the most complicated and ubiquitous posttranslational process found on a variety of secretory and membrane- bound proteins. Related enzymes, glycosyltransferases, are almost entirely specific to endoplasmic reticulum (ER), Golgi, and the extracellular milieu., most likely due to differential glycosyltransferase expression or activity. As a result, aberrant 0linked glycosylation has been extensively demonstrated to affect biological function and protein integrity that result in cancer growth and progression of several other diseases [28].

Disseminated neoplastic cells can undergo a period of dormancy but often grow and hijack the bone microenvironment. Eventually, in breast cancer the bone is the most common metastatic site. Adipocytes, one of bone marrow cell types, affect neoplastic cell proliferation by the expression of leptin, adiponectin, and tumor necrosis factor $\alpha$ and interleukin 6. The neoplastic cells support their growth by secreting vascular endothermal growth factor, parathyroid hormone related protein, matrix metalloproteinase, and interleukins that promote receptor activator of nuclear factor kappa- $\beta$-ligand [29].

Cluster of Differentiation (CD) 38 is a multifunctional ecto-enzyme that is the main nicotinamide dinucleotide (NAD+) catabolic enzyme. In addition, CD38 is an emerging therapeutic target under conditions in which metabolism is altered including infection, aging, and tumorigenesis. Thus, the therapeutic approaches to CD38 inhibition may serve as a treatment option for age-related metabolic dysfunction and tumor immunometabolism. CD38 is predominantly expressed on immune cells and metabolizes NAD to adenosine diphosphate ribose and cyclic adenosine diphosphate ribose which results in the mobilization of calcium. Most of CD38 activity takes place in extracellular domain which results in degradation of NAD+precursors, for example, nicotinamide mononucleotide (NAM) necessary for NAD+synthesis, although a trace amount of CD38 is present in the cytoplasm and in the membranes of organelles. Extracellular activity of CD38 has a wide range of imprecations for NAD+homeostasis in the context of infection, aging and tumor biology [30]. (Thomas et al., 2020) stated that DNA methylation profiling of choroid plexus cancer will aid the understanding of choroid plexus tumor biology, and can not only be used for the 
patient identification at risk of recurrence, but also play an important role for the treatment stratification and the patient management [31].

As far as the mechanism of epigenetic modifications is concerned, the modification can be grouped into three categories: Histone modifications, DNA and RNA methylations, and non-coding RNA manipulations. Notable examples of FDA approved epigenetic drugs, so-called epidrugs, include histone deacetylase (HDAC) inhibitor, DNMT inhibitor, and non-coding RNA (ncRNA) targeted drug. The first group constitutes volinostat, panobinostat, belinostat, and romidepsin, the second group, azacytidine and decitabine which are cytosine analogue inhibitor, and the third group, patistiran [32], volinostat, panobinostat, belinostat, and romidepsin. Other FDA approved epidrugs are azacytidine which is DNMT inhibitor and 5aza-2'-deoxycytidine which is cytidine analog. HDAC inhibitors act by blocking the catalytic domain of HDACs and are related to changes in the acetylation patterns of histones [33].

Docking research was initiated by (Kuntz ID et al., 1982) [34] and thereafter a series of docking systems have been introduced [35]. Among them, AutoDock is one of the most popular systems and utilized by many researchers [36]. Originally, all docking systems had to pursue one compound at a time which requires a sufficient amount of time. Although it depends on the system one is working on, docking of one compound requires, roughly speaking, one hour or so for a beginner, and half an hour or so for a regular user in order to accomplish a satisfactory docking of one compound. For a project to search for a safe and effective drug candidate, thousands of compounds have to be docked which needs a tremendous amount of time. To overcome this cumbersome process a virtual screening system has come into the place along with huge compound databases [37] such as ZINC, and ChemBridge [38].

Marine natural products (MNP) have been identified as a drug source for cancer treatment. (Ruiz-Torris V et al., 2017) reported an overview of MNP natural products and described their putative mechanisms of action [39]. Along with this overview, they examined the use of virtual screening for the aid of drug discovery from MNP in origin and introduced a novel and open-access chemical database.
It is claimed that due to the resistance emergence against antimalarial drugs, new antimalarial scaffolds are crucial to avoid antimalarial cross resistance [39]. They took advantage of the PyRx virtual screening system for the ZINC database search in order to discover resistant-free antimalarial drugs. The severe acute respiratory syndrome COVID 19 spreads around the world and poses a serious global concern not only from the standpoint of morbidity but also from the avoidance of mortality. To date, no compelling therapeutic agent is available to treat COVID 19. The inhibition of proteases essential for proteolytic processing of viral polyproteins is said to be a conventional therapeutic strategy. (Fischer A et al., 2020) conducted virtual screening of over 606 million compounds for binding at crystal structure of the main protease (Mpro) of COVID 19 [40]. Virtual screening is one of the hot topics in the area of research and development of drug discovery. VSDK, which is a technological VS system, was reported by (Baba E et al., 2011) [41]. It has a function to search a drug candidate from compound databases for a particular disease which is caused by a specific protein involvement.

\section{METHODS AND MATERIALS}

\section{Virtual screening experiment}

For the current VS experiment, VSDK [41] was used. Macromolecules used for this virtual screening were two epigenetic drug targets, HHEM and DNMT. The first step involved in the virtual screening is; a) Create the virtual screening file named VSfile (user's arbitrary name can be used) in Windows platforms, b) Download the epigenetic target protein from Protein Data Bank [ 42], convert them to pdbqt format by AutoDockTool [36], and then save it in VSfile. c) Download at random small molecules for the amount of 2778 from ZINC database [43], and convert them to pdb format by Facio [44] which is again converted to pdbqt format by AutoDockTool, and then save them in VSfile. The second step is; a) Create conf. txt as shown in fig. 1 and saved it in VSfile, b) Write the bash script as shown in fig. 2 and store it in VSfile, c) Open Cygwin64 terminal and execute VS01. bash command for virtual screening of the data, d) Transfer the data to Excel and sort them in the ascending order so that the one with the lowest affinity appears first.

Conf.txt file for HHEM (pdb code: 5YOD)

All

receptor $=5 Y 0 D \_l o c k \_$rigid.pdbqt $\quad \leftarrow$ receptor $($ macromolecule $)$ file

The detailed description of Conf.txt file for histone DNA methylase (pdb code: 5Y0D) used for virtual screening

All

receptor $=5$ Y0D_lock_rigid.pdbqt $\leftarrow$ receptor $($ macromolecule) file

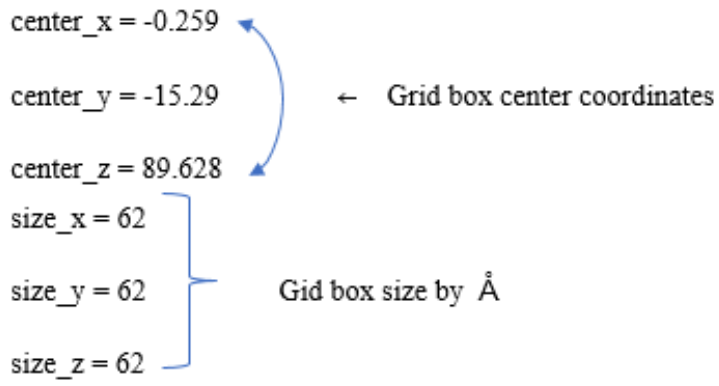

Fig. 1: The detailed description of conf. txt file for the VS of HHEM (pdb code: 5YOD) used for virtual screening from 2778 small ZINC molecules randomly retrieved 
-\#!/bin/bash

- for $f$ in ZINC*.pdbqt; do $b=$ 'basename $\$ f$.pdbqt'; echo Processing ligand $\$ b$; mkdir -p data01; . Vina/vina --config conf.txt --ligand \$f --out data01/\$f.pdbqt --log data01/Sf.txt; done

- \#result analysis

- cd data01

- grep " 1 "*.txt | cut -c1-12,35-42 >>result

- cat result

Fig. 2: The detailed script of bash file for HHEM used for virtual screening based on 2778 ZINC small molecules randomly retrieved

\section{Docking experiment}

AutoDock4, which is developed by Olson's group (Olson A et al., 2004) [36], is a computational application to achieve an optimized conformation between a target protein and a ligand so that the affinity $(\mathrm{kcal} / \mathrm{mol})$ between them is minimized. It is a powerful computational tool to reduce the labor and cost of the drug development. One can choose either the generic algorithm or the simulated annealing in AutoDock 4.

The computer used for the docking experiment is Fujitsu Lifebook Windows 10 . The grid box encompassing the binding site, where the ligand was embedded, was created and the grid maps representing the co-crystallized ligand and the receptor protein (pdb code: 5y0d and 4wxx) were pre-calculated using the AutoGrid (included in AutoDock package). The grid box was chosen to be sufficiently large enough to engulf not only the active site but also the pertinent portion of the surrounding surface. The size of grid box was thus designated to be $60 \times 60 \times 60$ in $\AA$ with a grid spacing of $0.375 \AA$. Since the location of embedded ligand in the complex is known, the cubic grid box was centered on the pivot of the embedded ligand binding site. After the AutoGrid process culminated in the successful completion, AutoDock was run to calculate the binding free energy between the given inhibitor and the receptor protein. The Genetic

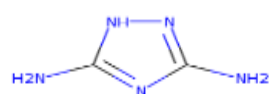

1. Z04118774; M: 99

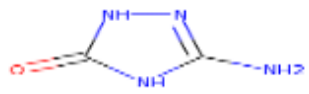

2. Z05425396; M: 96

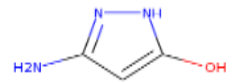

3. Z03591800; M: 100

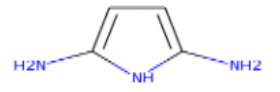

4. Z16970667; M: 97

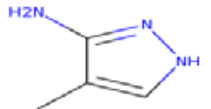

5. Z35655479; M: 97

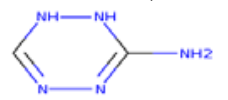

6. Z38700903; M: 100

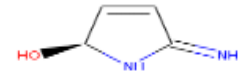

7. Z01576330; M: 99

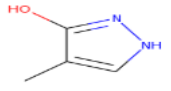

8. Z03861216; M: 98

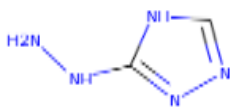

9. Z05522092; M: 99

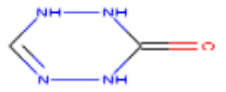

10. Z05543785; M: 100

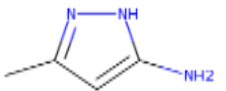

11. Z03886974; M: 98

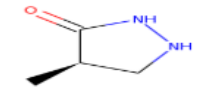

12. Z05758018;M: 100

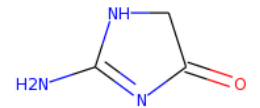

13. Z05838989; M: 99

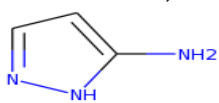

14. Z08616249: MW: 84

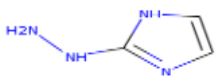

15. Z08628559; M: 98

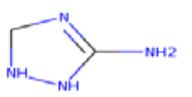

16. Z05758018; M: 87

Algorithm with Local Search (GALS) was applied for the interaction/binding mode between the receptor protein and the inhibitor. Cluster analysis was performed on the docking run applying Root Mean Square (RMS) with tolerance of $0.5 \AA$. The top ten clusters were ranked from the average lowest energy to the highest.

\section{RESULTS AND DISCUSION}

The first group of this experiment includes: a) Virtual screening results produced a sizable difference of binding energy between the top and bottom molecules with the magnitude of 2.4 times, b) As far as molecular types of the top ten compounds of HHEM inhibitors are concerned, eight compounds showed 5-member ring structures including triazole, triazol, pyrazoline, pyrrolidine, and pyrazol. The second group includes: a) DNMT inhibitors show 5-member ring structures just as in HHEM inhibitors along with cyclopentanone and cyclopentane as characteristic molecular structures, b) The VS search was successfully competed in eight h $20 \mathrm{~min}$ for HHEM inhibitors and ten h 10 min for DNMT inhibitors, c) The docking experiment represented the satisfactory outcome for all three criteria including hydrogen bond creation, the highest peak in the lower affinity region in the cluster histogram, and the favorable docking energy in the lower affinity region.

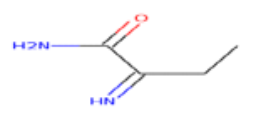

17. Z28766733; M: 100

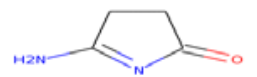

8. Z32599192; M: 98

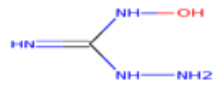

19. Z43278608; M: 91

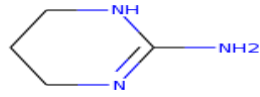

20. Z01728936; M: 100

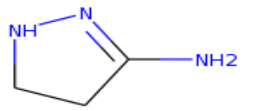

21. Z02579084; M: 86

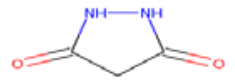

22. Z05382437; M: 100

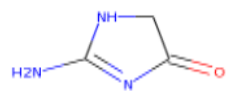

23. Z13536079; M: 91<smiles>NNC1NCCCN1</smiles>

24. Z13746443; M: 100

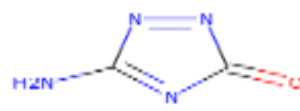

25. Z38520487; M: 98

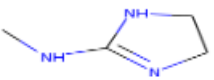

26. Z01605495; M: 100

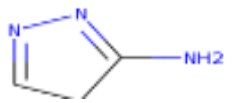

27. Z04674340; M: 84

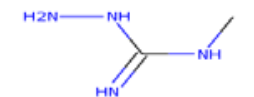

28. Z04804787; M: 89

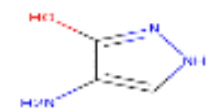

29. Z05500372; M: 100

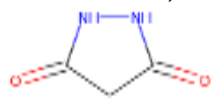

30. Z06495192; M: 100

Fig. 3: ZINC (Z) molecular structures of the top 30 compounds in terms of affinity (Kcal/mol) obtained by VSDK virtual screening for HHEM with moeculer weight (M) 


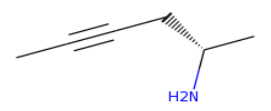

2769. Z1665159;M: 97

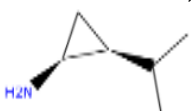

2770. Z51690491;M: 100

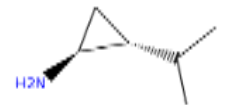

2771. Z51690492;M: 100

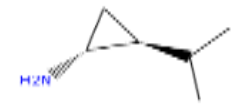

2772. Z51690498; M: 100

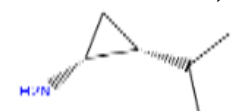

2773. Z51690494;M100

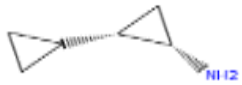

2774. Z51690975;M: 98

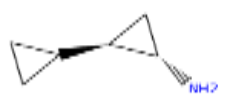

2775. Z51690997;M: 98

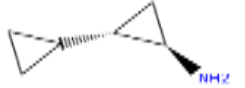

2776. .Z51690978;M: 98

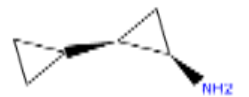

2777. Z51690980;M: 98

Fig. 4: ZINC (Z) molecular structures of the bottom 10 compounds in terms of affinity (Kcal/mol) obtained by VSDK VS for H HEM with molecular weight (M)

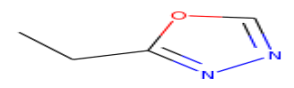

1. Z15423457;M: 98

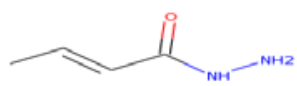

2. Z01596393;M: 100

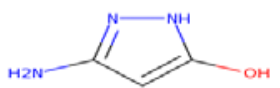

3. Z03591800; M: 100

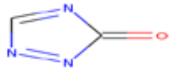

4. Z05382426; M: 83

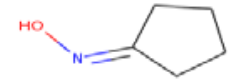

5. Z00967469;M: 99

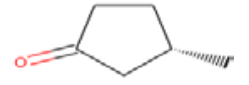

6. Z02039266;M: 98

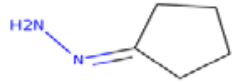

7. Z01576330; M: 99

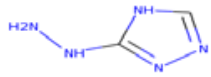

8. Z05522092;M: 99

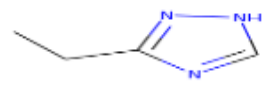

9. Z05688507;M: 97

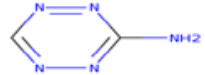

10. Z08251252;M: 97

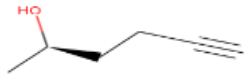

11. Z43656671; M: 98

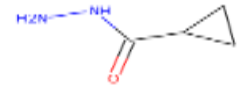

12. Z01696497;M: 100

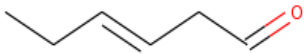

13. Z018433472; M: 98

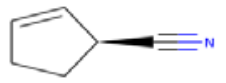

14. Z02032340: M: 93

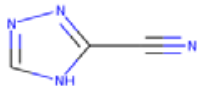

15. Z04218567; M: 93

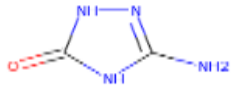

16. Z05425396; M: 100

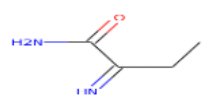

17. Z05638792; M: 100

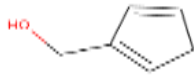

18. Z12358631; M: 96

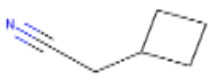

19. Z19093253; M: 95

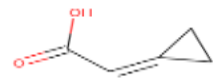

20. Z20997971; M: 97

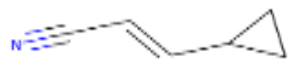

21. Z51690995; M: 93

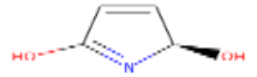

22. Z01607934; M: 99

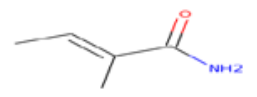

23. Z01763170; M: 99

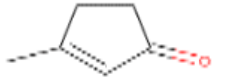

24. Z02035915; M: 96

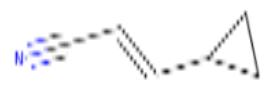

2778. Z51690995;M: 98

Fig. 5: ZINC (Z) molecular structures of the top 30 molecules out of ZINC 2778 compounds in terms of affinity (kcal/mol) obtained by VSDK VS for DNMT with molecular weight (M)

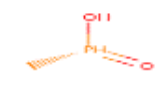

2769. Z14880500;M: 79

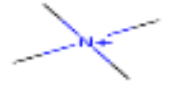

2770. Z00967771;M: 74

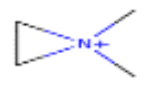

2771. Z06068236; M: 72
2772. Z12358605;M: 46

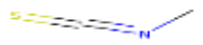

2773. Z08830589;M: 73

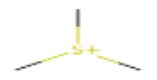

2774. Z08216665;M: 77
2775. Z0822105;M: 94 2776. Z15633213;M: 98 This compound is no longer available inZINC

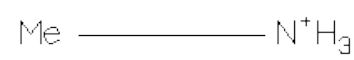

2777.Z15633215;M: 32

Fig. 6: ZINC (Z) molecular structures of the bottom 10 molecules out of ZINC 2778 compounds in terms of affinity (Kcal/mol) obtained by VSDK VS for DNMT with molecular weight (M) 
Table 1a: Virtual screening result of $1 \mathrm{wxx}$, DNA methylator, against ZINC compounds by using VSDK: top 30 ZINC drug-like molecules based on the affinity (Kcal/mol) out of 2778 compounds

\begin{tabular}{|c|c|c|c|c|c|c|c|c|}
\hline No & ZINC number & $\begin{array}{l}\text { affinity (Kcal/ } \\
\text { mol) }\end{array}$ & NO & ZINC number & $\begin{array}{l}\text { affinity } \\
\text { (Kcal/mol) }\end{array}$ & NO & ZINC number & $\begin{array}{l}\text { affinity (Kcal/ } \\
\text { mol) }\end{array}$ \\
\hline 1 & ZINC15423457 & -5.8 & 11 & ZINC43656671 & -5.4 & 21 & ZINC51690995 & -5.3 \\
\hline 2 & ZINC01596393 & -5.7 & 12 & ZINC01696497 & -5.3 & 22 & ZINC01607934 & -5.2 \\
\hline 3 & ZINC04582838 & -5.5 & 13 & ZINC01843472 & -5.3 & 23 & ZINC01763170 & -5.2 \\
\hline 4 & ZINC05382426 & -5.5 & 14 & ZINC02032340 & -5.3 & 24 & ZINC02035915 & -5.2 \\
\hline 5 & ZINC00967469 & -5.4 & 15 & ZINC04218567 & -5.3 & 25 & ZINC02039394 & -5.2 \\
\hline 6 & ZINC02039266 & -5.4 & 16 & ZINC05425396 & -5.3 & 26 & ZINC03591800 & -5.2 \\
\hline 7 & ZINC04887228 & -5.4 & 17 & ZINC05638792 & -5.3 & 27 & ZINC05133329 & -5.2 \\
\hline 8 & ZINC05522092 & -5.4 & 18 & ZINC12358631 & -5.3 & 28 & ZINC05133539 & -5.2 \\
\hline 9 & 9.INC05688507 & -5.4 & 19 & ZINC19093253 & -5.3 & 29 & ZINC05426776 & -5.2 \\
\hline 10 & ZINC08251252 & -5.4 & 20 & ZINC21997971 & -5.3 & 30 & ZINC06661263 & -5.2 \\
\hline
\end{tabular}

Table 1b: Virtual screening result of 1wxx, DNA methylator, against ZINC compounds by using VSDK: top 10 ZINC drug-like molecu; les based on the affinity (Kcal/mol) out of 2778 compounds

\begin{tabular}{lll}
\hline NO & ZINC number & Affinity (Kcal/mol) \\
\hline 1 & ZINC14880500 & -2.7 \\
2 & ZINC00967771 & -2.6 \\
3 & ZINC06068236 & -2.6 \\
4 & ZINC12358605 & -2.6 \\
5 & ZINC08830539 & -2.5 \\
6 & ZINC08216665 & -2.2 \\
7 & ZINC08221057 & -2.1 \\
8 & ZINC15633213 & -2.1 \\
9 & ZINC15633215 & -2 \\
10 & ZINC25783202 & -2 \\
\hline
\end{tabular}

\section{VS feature}

One of the docking methods is to explore geometrically feasible alignments of ligands and a receptor of known structure. (Kozakov et al., 2005) reported a geometric approach toward macromolecule ligand interactions and asserted that their approach seems to a wellsuited search generating conformations for energy refinement and interactive computer graphics routines [23].

With respect to the virtual screened result of HHEM, the top 30 molecules in terms of affinity are listed in fig. 3. The first rank molecule is $1 \mathrm{H}-1,2,4$-triazole-3,5-diamine. The average molecular weight of these top 10 molecules is 99 . The bottom ten molecules are listed in fig. 4. The average molecular weight of these 10 molecules is 98. As far as the size of the molecular weight is concerned not much difference is observed between the two groups. The affinity table for the top 30 molecules is shown in table 1a, and for the bottom ten, in table $1 \mathrm{~b}$. The average affinity for the top ten molecule is $-5.8 \mathrm{Kcal} / \mathrm{mol}$, and that for bottom ten, $-2.4 \mathrm{Kcal} / \mathrm{mol}$. The average affinity for the top ten molecule was 2.4 times greater than that of the bottom ten molecules, and this is a significant outcome, which indicates in a sense that the virtual screening for HHEM against 2778 ZINC molecules operated producing a sizable difference between the strong and weak inhibitors. This fact on affinity difference also helps find out a safe and an effective compound in the drug development strategy.

As to the experimental result performed for the virtual screened of DNMT the top 30 molecules in terms of affinity are listed in fig. 5 . The first ranked molecule is 2-ethyl-1,3,4-oxadiazole. The average molecular weight of these top ten molecules was 97 . The bottom ten molecules are listed in fig. 6 . The average molecular weight of these 10 molecules was 68. Roughly speaking, the average molecular weight of the bottom ten molecules is two thirds of that of the top ten molecules. The affinity value for the top 30 molecules is shown in table $2 \mathrm{a}$, and for the bottom ten, in table $2 \mathrm{~b}$. The average affinity for top ten molecule is $-5.5 \mathrm{Kcal} / \mathrm{mol}$, and that for the bottom ten,-2.3 $\mathrm{Kcal} / \mathrm{mol}$. The average affinity for the top ten molecule was 2.4 times greater than that of the bottom ten molecules. This is a valuable piece of information which was obtained by the virtual screening. As for DNMT inhibitors the average affinity for the top ten molecules is$5.49 \mathrm{Kcal} / \mathrm{mol}$, and that for the bottom ten, $2.34 \mathrm{Kcal} / \mathrm{mol}$. The average affinity for the top ten molecule was 2.3 times greater than that of the bottom ten molecules. It is unambiguously elucidated that our virtual screening of 2778 ZINC molecules was ranked from the one with strong inhibition to weak inhibition with an explicit difference. Here I propose that one of the criteria to judge the success of the virtual screening is "more than 2 times" difference in the average affinity between the top 10 inhibitors and the bottom ten inhibitors.

Table 2a: Virtual screening result of 5Y0D, histone deacetylase, by using VSDK; top $30 \mathrm{ZINC}$ drug-like molecules out of 2778 compounds

\begin{tabular}{lll}
\hline NO & ZINC number & Affinity (Kcak/mole) \\
\hline 1 & ZINC04118774 & -6.0 \\
2 & ZINC05425396 & -5.9 \\
3 & ZINC03591800 & -5.8 \\
4 & ZINC16970667 & -5.8 \\
5 & ZINC35655479 & -5.8 \\
6 & ZINC38700903 & -5.8 \\
7 & ZINC01576330 & -5.6 \\
8 & ZINC03861216 & -5.6 \\
9 & ZINC05522092 & -5.6 \\
10 & ZINC05543785 & -5.6 \\
11 & ZINC03886974 & -5.5 \\
12 & ZINC05758018 & -5.5 \\
13 & ZINC05838989 & -5.5 \\
14 & ZINC08616249 & -5.5 \\
15 & ZINC08628559 & -5.5 \\
16 & ZINC16778243 & -5.5 \\
17 & ZINC28766733 & -5.5 \\
18 & ZINC32599192 & -5.5 \\
19 & ZINC43278608 & -5.5 \\
20 & ZINC01728936 & -5.4 \\
21 & ZINC02579084 & -5.4 \\
22 & ZINC05382437 & -5.4 \\
23 & ZINC13536079 & -5.4 \\
24 & ZINC13746443 & -5.4 \\
25 & ZINC38520487 & -5.4 \\
26 & ZINC01605495 & -5.3 \\
\hline
\end{tabular}




\begin{tabular}{lll}
\hline 27 & ZINC04674340 & -5.3 \\
28 & ZINC04804787 & -5.3 \\
29 & ZINC05500372 & -5.3 \\
30 & ZINC06495192 & -5.3 \\
\hline
\end{tabular}

As far as molecular types of the top ten compounds of HHEM inhibitors are concerned, eight compounds show 5-member ring structures including tiazole (rank \#1), triazol (rank \#2, 9), pyrazoline (rank \#3, 7), pyrrolidine (rank \#4), and pyrazol (rank \#5, 8). Therefore, it is suggested that the HHEM inhibitors are preferred to contain certain types of 5-member ring such as triazole and triazol as shown in fig. 3. On the other hand, DNMT inhibitors represent a little different molecular structure although eight compounds show 5-member ring structure just as in HHEM inhibitors. As a characteristic molecular structure, cyclopentanone (rank \#5, 6) and cyclopentane (rank \#7) are noticed. The other 5-member ring compounds include oxysadiazole (rank \#1), pyrazolone (rank \#3), trizol (rank \#4,8), and triazole (rank \#9) as shown in fig. 5. It is worth keeping in mind that the type of chemical structure is important to develop an innovative marketable drug. This process is necessary in research and development of all areas of drug categories such as anticancer drugs, anti-inflammatory drugs, antibiotics, and so on.

Table 2b: Virtual screening result of 5Y0D, histone deacetylase, by using VSDK; last 30 ZINC drug-like molecules out of 2778 compounds

\begin{tabular}{lll}
\hline NO & ZINC number & Affinity (Kcal/mol) \\
\hline 2769 & ZINC51665159 & -2.6 \\
2770 & ZINC51690491 & -2.6 \\
2771 & ZINC51690492 & -2.5 \\
2772 & ZINC51690493 & -2.5 \\
2773 & ZINC51690494 & -2.5 \\
2774 & ZINC51690975 & -2.5 \\
2775 & ZINC51690977 & -2.5 \\
2776 & ZINC51690978 & -2.1 \\
2777 & ZINC51690980 & -2.1 \\
2778 & ZINC51690995 & -2.0 \\
\hline
\end{tabular}

\section{AutoDock verification}

(Zhang X et al., 2020) conducted a research on. Biological, clinical and epidemiological features of COVID-19, SARS and MERS, and AutoDock simulation of ACE2, which is up-to-date topic worldwide.[45]. (Kozakov et al., 2005) reported a geometric approach to macromolecule ligand interactions. Their method is to explore geometrically feasible alignments of ligands and receptors of known structure. They declared that their approach seems well-suited generating conformations for energy refinement programs and interactive computer graphics routines $[23,46]$. On the other hand, Goodsell et al. developed AutoDock which is compiled by C language used to predict the bound conformations of a small, flexible ligand against a macromolecule target of known structure for the search of the best-fit conformation with a rapid grid-based method of energy evaluation, and a simulated annealing technique was adapted [18]. AutoDock is one of the most valuable docking systems and has been used by quite a few numbers of researchers for the research and development of innovative drugs [47-50].

With respect to the evaluation of the docking result, the prominent criteria to apply for the selection of valuable compounds are considered to be the low free energy, the cluster appearance in the lower free energy region, and the creation of hydrogen bonds. The method AutoDock adopts for detecting the cluster is to measure the reliability of the docked result by comparing root mean squire deviation (RMSD) of the lowest energy conformation and grouping them into families of similar conformations or "clusters" [51]. With respect to protein docking, the underlining principle is that clustering occurs because long-range electrostatic and/or desolvation forces steer the protein to low free energy attractor at the binding region [52]. By default, AutoDock clusters the docked conformations at $2 \AA$ [50]. Generally speaking, the AutoDock result is considered to be favorable when the greatest cluster appears in the region of the lowest binding energy.

\section{The reliability of AutoDock}

The examples of AutoDock use for the search of innovative drugs amount to be substantial in number. PubMed retrieves 1801 articles [51] and Google Scholar, 70500 [52], as of May 31, 2021. Ali et al., reported the antitumor drug development study of novel fravin analogs by applying an AutoDock for a substantial study against protein tyrosine kinase pp60c-src along with an inhibitory experiment against tumor cell lines represented by $\mathrm{IC}_{50}(\mu \mathrm{M})$, where a good correlation between their $\mathrm{IC}_{50}$ and AutoDock binding free energy was validated. The histones epigenetic modifications are versatile marks that are intimately connected to the development of disease pathogenesis including human neoplasms [53]. Legitimate mutations of histone H2B are frequently found in cancer cells. Extrinsic production of the H2B E76K mutant ruggedly promote the colony formation ability of the expressing cells, elucidating the fact that the histone mutation has a potential to promote carcinogenic transformation [54]. (Yan et al., 2020) performed AutoDock calculations to determine and compare the binding affinities of remdesivir, chloroquine, ciclesonide, niclosamide, and lopinavirus to angiotensin-converting enzyme 2 (ACE2), which is considered to play a vital role in the development of COVID-19, and found that chloroquine, and ciclesonide share the similar amino acid residues such as leucine, methionine, Valine, and Isoleucine [55].

\section{Cluster and histogram}

The histogram chart of AutoDock4 100 runs of HHEM against 2778 ZINC compounds is shown in fig. 7 in which the greatest cluster appeared in the lowest region whose mean binding energy is-3.80 Kcal/mol. This indicates that this docking run completed with a resealable result which elucidates a proper docking execution. Fig. 8 shows the histogram chart of AutoDock4 100 runs of DNMT against 2778 ZINC compounds. This histogram chart also represents the greatest cluster appeared in the lowest energy region with mean binding energy of-3.80 kcal/mol. Therefore, the AutoDock operation in this experiment proved the validity of the docking with a reliable result.

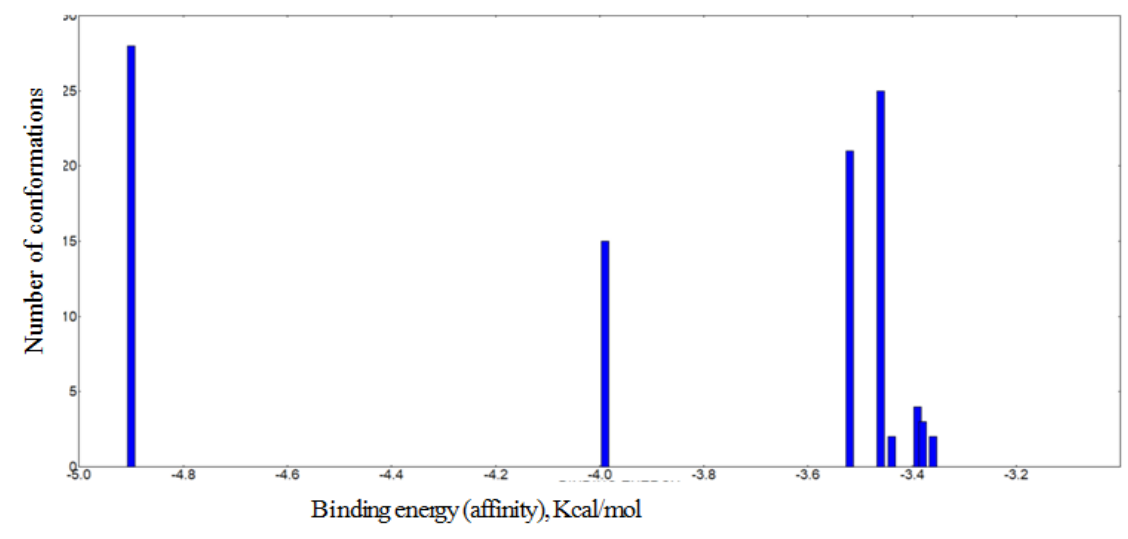


Fig. 7: Docking conformation cluster of the ZINC compound 1H-1,2,4-triazole-3,5-diamine against HHEM, showing the most tenacious affinity peak in the lowest region, obtained by AutoDock4 Tool [36]

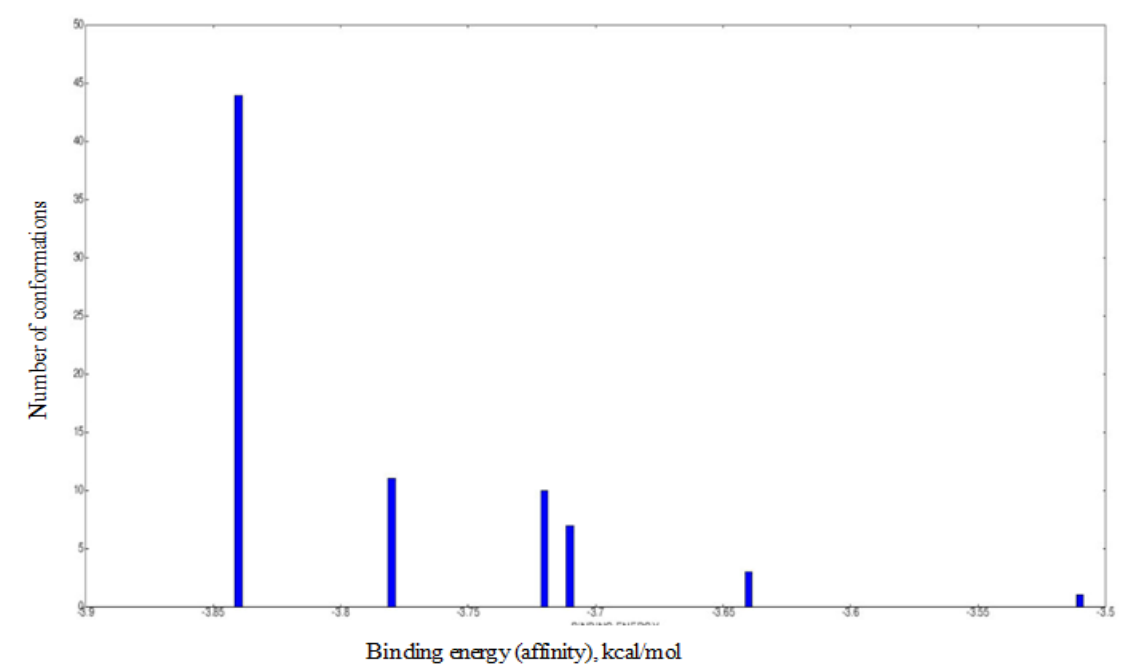

Fig. 8: Docking conformation cluster of the ZINC compound 2-ethyl-1,3,4-oxadiazole against DNMT showing the most tenacious affinity peak in the lowest region, obtained by AutoDock4 Tool [36]

(a)

(b)

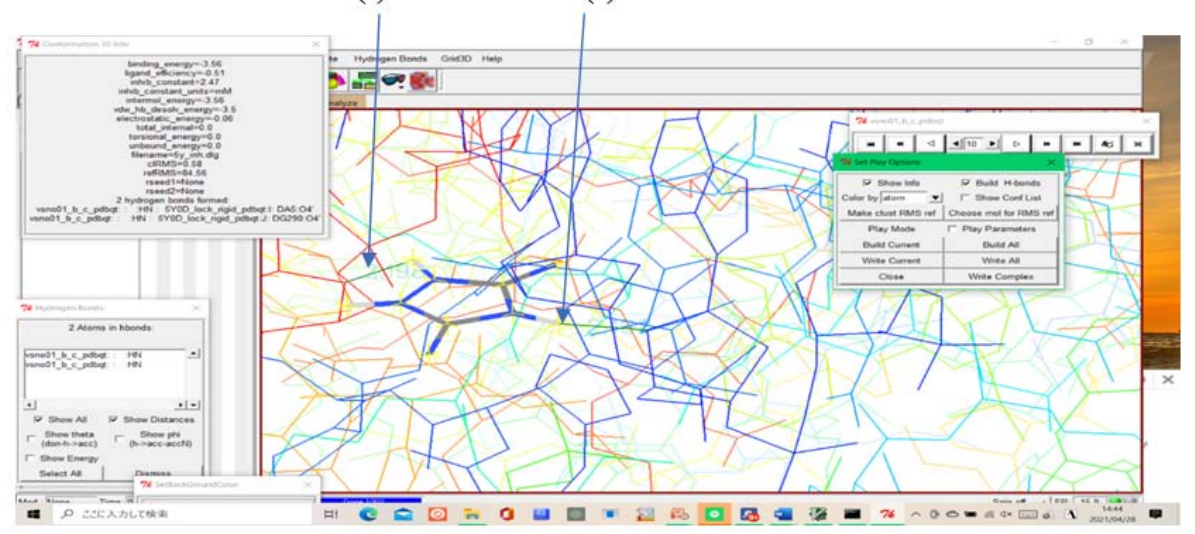

Fig. 9: The docking conformation of ZINCinhibitor against HHEM indicating the hydrogen band formed between the donor hydrogen of ZINC inhibitor and acceptor residue of HHEM; (a) hydrogen bond between ZINCinhibitor and HHEM D289 residue with the distance of 2.196

$\AA$, and (b) hydrogen bond between ZINCinhibitor and HHEM DS5 residue with the distance of 2.182, obtained by AutoDock4 Tool [36]

(a) (b)

(c)

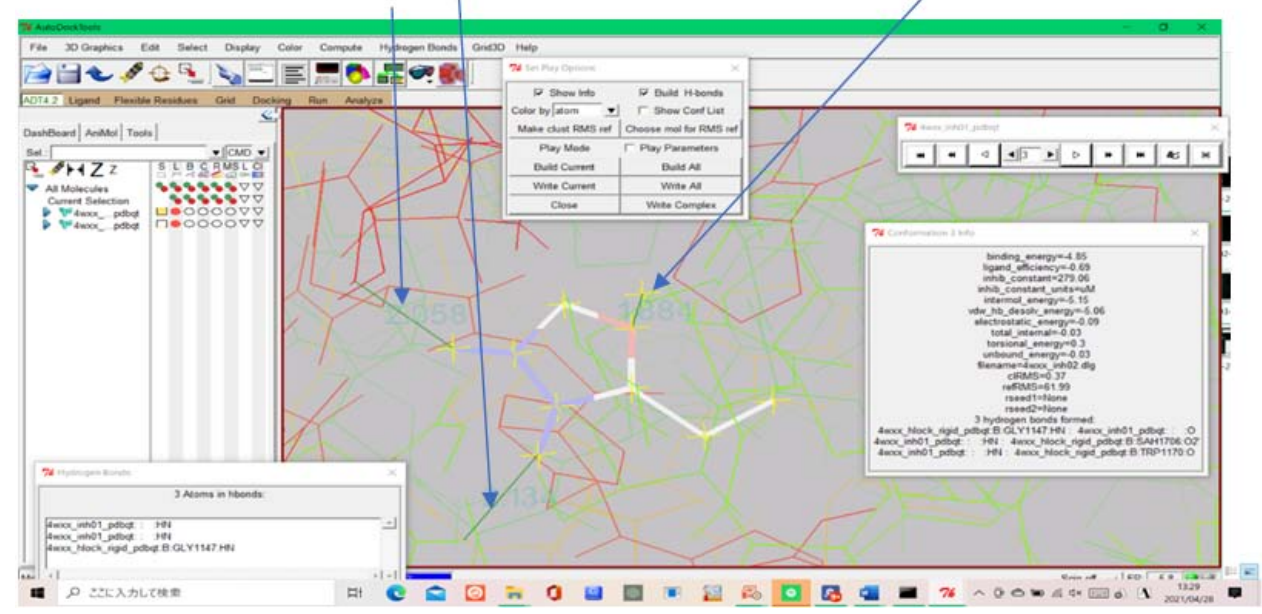

Fig. 10: The docking conformation of ZINC inhibitor against DNMT indicating the hydrogen band formed between the donor hydrogen of ZINC inhibitor and acceptor residues of DNMT; (a) hydrogen bond between the donor hydrogen of ZINC inhibitor and HHEM residue SAR1706 with the distance of $2.058 \AA$, (b) hydrogen bond between ZINC inhibitor and DNMT TRP1170 residue with the distance of 
$2.134 \AA$ and (c) hydrogen bond between the donor hydrogen of ZINC inhibitor and DNMT GRY1147 residue with the distance of 1.884 A, obtained by AutoDock4 Tool [36]

\section{Hydrogen bond}

As for the existence of hydrogen bond, two hydrogen bonds are formed between the best ZINC inhibitor and two HHEM residues as shown in fig. 9. The closer examination shows that the hydrogen bonds between two donor hydrogens of the ZINC inhibitor and acceptor oxygens of HHET DG289 and DA5 residues with the distance of $2.196 \AA$ and $2.182 \AA$ are observed. As shown in fig. 10. Three hydrogen bonds are formed between the donor hydrogen of the ZINC inhibitor and DNMT residues. Exploring one by one, hydrogen bond between the donor hydrogen of ZINC inhibitor and HHEM acceptor residue SAR1706 with the distance of $2.058 \AA$, the donor hydrogen of ZINC inhibitor and DNMT residue TRP1170 with the distance of $2.134 \AA$, and the donor hydrogen of ZINC inhibitor and HHEM residue GRY1147 with the distance of $1.884 \AA$

(Riza H et al., 2018) reported an inhibitor docking study of methyl hesperidin as nucleoside reverse transcriptase by using AutoDock Vina [56]. They reported a good result indicating the binding energy and biding site. However, they did not indicate the hydrogen bond creation nor the binding conformation histogram. (Chellam J et al., 2016) reported a computational biological study of aromatase inhibitors docking with human placental aromatase cytochrome P450 [57]. They have obtained internal energy of- $9.759 \mathrm{Kcal} / \mathrm{mol}$ which is excellent, although neither hydrogen bond formation nor cluster histogram are indicated. (Govindarasu M et al., 2017) presented docking studies on kaempferitrin in inflammatory and apoptosis proteins for the search of colon cancer treatment. It would be more convincing if they included more than one chemical [58].

(Tanisa AN et al., 2017) performed virtual screening study on betasecretase inhibitors using AutoDock and AutoDock Vina, and reported that they obtained six satisfactory compounds by AutoDock and seven by AutoDock Vina [59]. However, no number of compounds tested and no time spent for virtual screening were reported and it is hard to understand the details of their virtual screening.

In the pharmaceutical research field, we now come up with various types of docking and virtual screening research works such as understanding of structural interactions of protein and ligand [60], drug design and discovery [61], and docking against phytochemical screening, which all contribute to the enhancements of not only the pharmaceutical research productivity but also academic authenticity $[62,63,64]$. I am glad to see that this work is one of them and I wish this type of docking and virtual screening research would continue to be prosperous and productive in the pharmaceutical sciences.

\section{CONCLUSION}

For the 2778 compounds retrieved from the ZINC database, the virtual screening to search for the HHEM inhibitors was successfully competed in eight h $20 \mathrm{~min}$, and for the DNMT inhibitor, ten h ten $\min$. As for the molecular structure, eight compounds out of the top ten HHEM inhibitors contained 5-member rings such as triazole, triazol, pyrazoline, pyrrolidine, and pyrazol. In addition to 5member ring the top ten DNMT inhibitors contained cyclopentanone and cyclopentane. We propose that more than two times affinity difference between the top and bottom ten compounds indicate a successful outcome of the virtual screening experiment. The histogram chart of AutoDock 4100 runs of HHEM inhibitors against 2778 ZINC compounds appeared in the lowest region with the largest clusters for both HHEM and DNMT inhibitors. As for the existence of hydrogen bond, two or three hydrogen bonds are formed between the best ZINC inhibitor and HHEM or DNMT residues. Therefore, the AutoDock operation in this experiment proved its validity.

\section{ABRIVIATIONS}

histone H2B E76K mutant: (HHEM), DNA methyltransferase: (DNMT), Endoplasmic reticulum: (ER), Bromodomain extra terminal: (BET), Un-folded protein response: (UPR), Bromodomain: (BRD), Histone: $(\mathrm{H})$, Bromodomain protein 4: (BRD4), Histone acetyltransferase: (HAT), Nicotinamide mononucleotide: (NAM), Inositol-requiring enzyme: (IRE), Cluster of differentiation: (CD), Nicotinamide dinucleotide: (NAD), Histone deacetylase: (HDAC), Marine natural products: (MNP), Tumor necrosis factor $\alpha$ : (TNF $\alpha$ ), Coronavirus: (COPID 19), Main protease: (Mpro), Virtual screening: (VS), Virtual screening by docking: (VSDK), Root mean square: (RMS), Root mean square deviation: (RMSD), Angiotensin-converting enzyme 2: (ACE2).

\section{ACKNOWLEDGEMENT}

None

FUNDING

Nil

\section{AUTHOR CONTRIBUTION}

All the work have been carried out by me.

\section{CONFLICT OF INTERESTS}

Declared none

\section{REFERENCES}

1. Sung H, Ferlay J, Siegel RL, Laversanne M, Soerjomataram I, Jemal A, et al. Global cancer statistics 2020:.GLOBOCAN estimates of incidence and mortality worldwide for 36 cancers in 184 countries. CA Cancer J Clin 2021;71:209-49.

2. Siegel LS, Miller KD, Jmal M. Cancer statistics. CA Cancer J Clin 2020;70:7-30.

3. Forli S, Olson A. A forcefield with discrete displaceable waters and desolvation entropy hydrated ligand docking. J Med Chem 2012;55:623-38.

4. Bray F, Ferlay J, Soerjamataram I, Siegel RL, Torre LA, Jemal A. Global cancer statistics 2018: GLOBOCAN estimates of incidence and mortality worldwide for 36 cancers in 185 counties. CA: Cancer J Clin 2018;68:394-424.

5. Trott 0 , Olson AJ. AutoDock Vina: improving the speed and accuracy of docking with a new scoring function, efficient optimization and multithreading. J Comp Chem 2010;31:466-61.

6. Singh R, Bassett E, Chakravarti1 A, Parthun M. Replicationdependent histone isoforms: a new source of complexity in chromatin structure and function. Nucl Acid Res 2018;46:8665-78.

7. Mu TW, Ong DST, Wang YJ, Balch WE, Yates JR, Segatori L, Kelly JW. Chemical and biological approaches synergize to ameliorate protein-folding diseases. Cell 2008;134:769-81.

8. Cohen E, Paulsson JF, Blinder P, Burstyn Cohen T, Du D, Estepa $\mathrm{G}$, et al. Reduced IGF-1 signaling delays age-associated proteotoxicity in mice. Cell 2009;139:1157-69.

9. Djajadikerta A, Keshri S, Pavel M, Prestil R, Ryan L, Rubinsztein DC. Autophagy Induction as a therapeutic strategy for neurodegenerative diseases. J Mol Biol 2020;432:2799-821.

10. Yanjun M, Hendershot LM. The role of the unfolded protein response in tumor development: friend or foe. Nat Rev Cancer 2005;4:966-77.

11. Hetz C, Chevet E, Harding HP. Targeting the unfolded protein response in disease. Nat Rev Drug Discovery 2013;12:703-19.

12. Shi W, Chen Z, Li L, Liu H, Zhang R, Cheng $Q$, et al. Unravel the molecular mechanism of XBP1 in regulating the biology of cancer cells. J Cancer 2019;10:2035-46.

13. Longley DB, Johnson PG. Molecular mechanisms of drug resistance. J Phathol 2005;205:275-92.

14. Sang J, Kulkarni K, Watson GM, Ma X, Craik DJ, Henriques ST, et al. Evaluation of cyclic peptide inhibitors of the Grb7 breast cancer target: small change in cargo results in large change in cellular activity. Molecules 2019;24:1-19.

15. Peng SB, Zhang X, Paul D, Kays LM, Gough W, Stewart J, et al. Identification of LY251924, a novel cyclic peptide CXCR4 antagonist that exhibits antitumor activities a solid tumor and 
breast cancer metastatic models. Mol Cancer Ther 2014;14:480-90.

16. Khan MA, Tania M, Fu J. Epigenetic role of thymoquinone: impact on cellular mechanism and cancer therapeutics. Drug Discovery Today 2019;24:2315-22.

17. Shi YX, Sheng DQ, Cheng L, Song XY. Current landscape of epigenetics in lung cancer: focus on the mechanism and application. J Oncol 2019;2019:1-11.

18. Dawson MA, Kouzarides T. Cancer epigenetics: from mechanism to therapy. Cell 2012;150:12-7.

19. Masui K, Harachi M, Webster K, Cavennee K, Mischel PS, Shibata N. Codependency of metabolism and epigenetics derives cancer progression: a review. Acta Histochem Cytochem 2020;53:1-10.

20. Clapiew CR, Iwasa J, Cairns BR, Peterson CL. Mechanisms of action and regulation of ATP-dependent chromatin-remodeling complexes. Nat Rev Mol Cell Bio 2017;18:207-422.

21. Fujisawa T, Filippakopoulos P. Functions of bromodomaincontaining proteins and their roles in homeostasis and cancer. Nat Rev Mol Cell Bio 2017;18:246-62.

22. Trivedi A, Mehrotra A, Baum CE, Lewis B, Basuroy T, Blomquist T, et al. Bromodomain and extra-terminal domain (BET) proteins regulate melanocyte differentiation. Epigen Chrom 2020;13:1-18.

23. Kozakov D, Clodfelter KH, Vakda S, Camacho CJ. Optimal clustering for detecting near-native conformations in protein docking. Biophys J 2005;89:867-75.

24. Niranos A, Cassaccia P. Bromodomains: translating the words of lysine acetylation into myelin injury and repair. Neurosci Lett 2017;625:4-10.

25. Strahl BD, Aills CD. The language of covalent histone modifications. Nature 2000;403:41-5.

26. Garcia Guede A, Vera O, Ibafiez-de-Caceres I. When oxidative stress meets epigenetics: implications in cancer development. Antioxidants 2020;9:1-26.

27. Shin DG, Bayarsaihan D. A novel epi-drug therapy based on the suppression of BET family epigenetic readers. Yale J Bio Med 2017;90:53-71.

28. Gupta R, Leon F, Rauth S, Batra SK, Ponnusamy MP. A systematic review on the implication of 0 -linked glycan branching and truncating enzymes on cancer progression and metastasis. Cells 2020;446:3-25.

29. Zarrer J, Haider MT, Smit DJ, Taipaleenmaki H. Pathological crosstalk between metastatic breast cancer cells and the bone microenvironment. Biomolecules 2020;10:1-20.

30. Hogan KA, Chini CS, Chini EN. The multi-faceted ectoenzyme CD38:.roles in immunomodulation, cancer, aging, and metabolic diseases. Front Immunol 2019;10:1-12.

31. Thomas C, Metrock K, Metrock K, Kodes U. Epigenic impacts upon prognosis and clinical management of choroid plexus tumors. J Neuro Oncol 2020;148:39-45.

32. Yuanjun L, Chan YT, Tan HY, Li S, Wang N, Feng Y. Epigenic regulation in human cancer: potential role of epi-drug in cancer therapy. Mol Cancer 2020;19:1-16.

33. Miranda Furtabo CL, Dos Santos Luciano MC, Silva Santos RD, Furtado GP, Moraes MO, Pessoa C. Epidrugs: targeting epigenetic marks in cancer treatment. Epigenetics 2019;14:1164-76.

34. Kuntz ID, Blaney JM, Oatley SJ, Langridge R, Ferrin TE. A geometric approach to macromolecule ligand interactions. J Mol Biol 1982;161:269-88.

35. Pagadala NS, Khajamohiddin Syed, Tuszynski J. Software for molecular docking: a review. Biophys Rev 2017;9:91-102.

36. Morris GM, Goodsell DS, Pique ME, Lindstrom WL, Huey R, Forli S, et al. AutoDock, AutoGrid, AutoDock Tool. Scripps Research Institute. Cal, USA; 2004.

37. Awale M, Jin X, Rymond JL. Stereoselective virtual screening of the ZINC database using atom pair 3D-fingerprints. J Chem Inf 2015;7:3.

38. Vidler LR, Fiippakopoulos P, Fedorov O, Picaud S, Martin S, Tomsett M, et al. Discovery of novel small-molecule inhibitors of BRD4 using structure-based virtual screening. J Med Chem 2013;56:8673-88.

39. Ruiz Torres V, Encinar JA, Herranz Lopez M, Perez Sanchez A, Galiano V, Enrique Barrajon Catalan E, et al. An updated review on marine anticancer compounds: the use of virtual screening for the discovery of small-molecule cancer drugs. Molecules 2017;22:1-37.

40. Fischer A, Sellner M, Niranjan S, Smiesco M, Lill MA. Potential inhibitors novel coronavirus protease identified by virtual screening of 606 million compound. Int J Mol Sci 2020;21:3626-43.

41. N Baba, E Akaho. VSDK: virtual screening of small molecules using AutoDock vina on windows platform. Bioinformation 2011;6:387-8

42. Protein Data Bank, RCSB PDB: Homepage; 2021.

43. ZINC. Available from: http://zinc15.docking.org/tranches/home/ [Last accessed on 18 May 2021]

44. Facio. Available from: http://zzzfield.sakura.ne.jp. [Last accessed on 18 May 2021]

45. Zhang $\mathrm{X}$, Huang $\mathrm{H}$, Zhuang $\mathrm{D}$, Nasser MI, Yang $\mathrm{M}$, Zhu P. Biological clinical and epidemiological features of COVID-19, SARS and MERS and AutoDock simulation of ACE2. Infect Dis Poverty 2020;9:3-11.

46. French CA, Miyoshi I, Kubonishi I, Grier HE, Perez-Atayde AR, Flecher JA. BRD4-NUT fusion oncogene: a novel mechanism in aggressive carcinoma. Cancer Res 2003;63:304-7.

47. Goodsell DS, Morris GM, Olson JA. Automated docking of flexible ligands; applications of AutoDock. J Mol Recognit 1996;9:1-5.

48. Ali HI, Tomita K, Akio E, Kawashima Y, Yamagishi T, Ikea H, et al. Antitumor studies - part II: structure-activity relationship study for flavin analogs including investigations on their in vitro antitumor assay and docking simulation into protein tyrosine kinase. Euro J Med Chem 2008;43:1376-89.

49. Ueda S, Tanaka S. AutoDock-GIST; Incorporating thermodynamics of active-site water into scoring function for accurate protein-ligand docking. Molecules 2016;21:1064-24.

50. Zarrer J, Haider MT, Smit DJ, Taipaleenmaki H. Pathological crosstalk between metastatic breast cancer cells and the bone microenvironment. Biomolecules 2020;10:1-20.

51. Morris GM, Lim Wilby M. Molecular docking. Methods in molecular biology. Humana Press: Totowa, NJ, USA; 2008. p. 365-82.

52. Kozamov D, Codfelter KH, Vajda S, Camacho CJ. Optimal clustering for detecting near-native conformations. Biophys J 2005;89:867-85.

53. Zhao Z, Shilatifard A. Epigenetic modifications of histones in cancer. Gen Bio 2019;20:1-16.

54. Arimura Y, Ikura M, Fujita R, Noda N, Kobayashi W, Horikoshi $\mathrm{N}$, et al. Cancer-associated mutations of histones H2B, H3.1 and H2A. Z1 affect the structure and stability of the nucleosome. Nucl Acid Res 2018;46:10007-18.

55. Yan R, Zhang Y, Li Y, Xia L, Guo Y, Zhou Q. Structural basis for the recognition of the SARS-COV-2 by full-length human ACE2. Science 2020;367:1444-8.

56. Riza H, Fahrurruji A, Wicaksono A, Nugroho AK, Martono S. Docking study of methyl hesperidin as nucleoside transcriptase inhibitor. Int J Pharm Pharm Sci 2018;10:85-9.

57. Chellam J, Deepai R. Computational biological study of aromatase inhibitors docking with human placental aromatase cytochrome p450. Int J Pharm Pharm Sci 2016;7:93-7.

58. Govindarasu M, Palani M, Vaiyapuri MI. In silico docking studies on kaempferitrin with diverse inflammatory and apoptotic proteins functional approach towards the colon cancer. Int J Pharm Pharm Sci 2017;9:199-204.

59. Tanisa AN, Riadhi R, Yanuar A. Virtual screening of betasecretase 1 (BACE 1) inhibitors in the Indonesian herbal database as using AutoDock and AutoDock Vina. Asian J Pharm Clin Res 2017;10:148-52.

60. Chahdrasekaran R, Sivaraman T, Sivaramakrishnan S, Kumar JJ. A computational approach on understanding structural interactions of envelope protein of dengue virus bound with squalene, a prototype anti-viral compound. Int J Pharm Pharm Sci 2019;11:1113-6.

61. Sharma A, Kunwar S, Agarwal V, Singh C, Sharma MD, Chauhan N. Molecular docking: an explanatory approach in structure-based drug designing discovery. Int J Pharm Pharm Sci 2021;13:6-12. 
62. Ibrahin H, Elsharawy FS, Elhassab M, Shabana S, Haggag G. Phytochemical screening and biological evaluation of Dypsis Leptocheilog leaves extract and molecular docking study of the isolated compounds. Int J Pharm Pharm Sci 2020;12:106-13.

63. Musfiroh I, Khatami HA, Megantara S, Muchtaridi M. Molecular dynamic simuation of asiatic acid derivatives complex with inducible nitric oxide synthase enzyme as an anti-inflammatory. Int J Appl Pharm 2021;13:32-8.

64. Pradeelp S, Shrungesh KT, Prashantha N, Majadevan KM. Synthesis in vitro antibacterial, toxicology and macromolecular docking anticancer activity of novel N-[(2-chloroquinolin-3-yl) methylidene]-2-anilin schiff bases. Int J Curr Pharm Res 2015;7:37-46. 